\title{
Aktuelle RCTs zu bewegungsthera- peutischen und psychomotorischen Interventionen zur Förderung der sozialen und emotionalen Entwicklung von Kindern
}

\author{
Iris Bräuninger
}

n diesem Beitrag werden zwei internationale RCTs (randomisierte kontrollierte Studien) vorgestellt, welche bewegungstherapeutische und psychomotorische Interventionen auf ihr Potential überprüften, durch Bewegung die emotionale und soziale Entwicklung bei Kindern mit Lernschwierigkeiten bzw. mit selektivem Mutismus zu fördern.

\section{Bewegungstherapie zur Förderung emotionaler und perzeptual-moto- rischer Fähigkeiten von Kindern mit \\ Lernschwierigkeiten}

Die Interventionsstudie von Alotaibi und KollegInnen (2017) wurde in Saudi-Arabien mit 6-9 Jungen im Grundschulalter $(\mathrm{N}=60)$ mit leichten Lernschwierigkeiten durchgeführt. Die Hypothese ging davon aus, dass die bewegungstherapeutische Gruppenintervention die emotionalen, sozialen und perzeptuell-motorischen Fähigkeiten der Kinder fördern würde. Die Kinder (mit IQ zwischen 50 und 70 Punkten) wurden auf eine Interventionsgruppe $(n=30)$ und eine Kontrollgruppe $(n=30)$ randomisiert. Beide Gruppen erhielten über acht Wochen hinweg dreimal wöchentlich entweder Bewegungstherapie als Intervention (Chace Ansatz) oder Sportunterricht als Kontrollgruppenbedingung. Im Vor-, Nachtest wurde die arabische Version des Purdue Perceptual-
Motor Survey durchgeführt, um die perzeptuell-motorischen Fähigkeiten anhand von Balance und Körperhaltung zu testen. Der Goodman's Strength and Difficulties Fragebogen (SDQ) zum Verhaltensscreening (Goodman 2000) wurde in der Fremderhebungsversion den Lehrern verabreicht. Die Gruppen waren vor der Intervention in Bezug auf Alter und Intelligenz vergleichbar (Durchschnittsalter Intervenion 8.1 Jahre (SD 0.86, Range 6-9), IQ 59.8 (SD 4.67, Range 50-66); Durchschnittsalter Kontrollgruppe 7.8 Jahre (1.19, Range 6-9) und IQ 58.5 (SD 5.62, Range 51-69). Alle Fragebögen wurden zur Vor- und Nachtestung ausgefüllt, und es gab keine Dropouts und keine Fehlzeiten. Die Ergebnisse zeigten im Posttest signifikante Korrelationen zwischen dem emotionalen Wohlbefinden der Kinder und der perzeptual-motorischen Fähigkeit nach dem achtwöchigen Bewegungstherapie-Programm; dies war in der Kontrollgruppe nicht der Fall. Die Ergebnisse könnten, so die AutorInnnen, ein Hinweis darauf sein, dass die Gruppenbewegungstherapie die Integration emotionaler, sozialer und perzeptuell-motorischer Fähigkeiten der Kinder förderte. 


\section{Psychomotorik zur positiven}

\section{Veränderung des Lebensstils von} Kindern mit selektivem Mutismus

Esposito und KollegInnen (2016) überprüften in einem RCT die Wirksamkeit einer sechsmonatigen psychomotorischen Standardbehandlung auf die positiven Veränderungen des Lebensstils von Kindern mit selektivem Mutismus (SM). „Selektiver Mutismus (SM) ist eine seltene Erkrankung bei Kindern, die von DSM-5 als Angststörung kodiert wurde. Trotz der beeinträchtigenden Natur der Krankheit gibt es noch keine spezifische Behandlung“ (Esposito et al, 2016, Übersetzung durch die Autorin). An der Studie nahmen insgesamt 138 Kinder teil, welche auf eine Psychomotorik-Intervention $(n=67)(M=35$, mittleres Alter $7,84 \pm 1,15)$ und eine Kontrollgruppe $(n=71)(M=37$, mittleres Alter $7,75 \pm 1,36$ ) randomisiert wurden. Die Intervention wurde über sechs Monate hinweg dreimal wöchentlich zu 45 Minuten im Wohngebiet der Kinder durch PsychomotoriktherapeutInnen durchgeführt. Die Eltern der Kontrollgruppe erhielten zu Beginn der Studie eine Verhaltens- und Bildungsberatung. Vor Beginn und am Ende der Behandlung wurden alle Kinder einer Verhaltensbewertung unterzogen (italienische Version der Child Behavior Checklist CBCL; Achenbach 1991), um die Wirksamkeit des psychomotorischen Managements zu überprüfen. Die Eltern füllten außerdem den Selektiven Mutismus Fragebogen SMQ aus (italienische Version; Bergman et al. 2008). Am Ende der sechsmonatigen Behandlung zeigten die Kinder der Psychomotorik-Intervention eine signifikante Verbesserung der psychischen Befindlichkeit in Bezug auf die Skalen „Social Relations“ ( $p<0.001)$, „School Competences“ $(p=0.005)$, Sozialer Rückzug ( $p<0.001)$, KörperlicheBeschwerden $(p=0.041)$, Angst/Depressivität ( $p<0.001)$, Soziale Probleme ( $p$ <.001), Denk-, (Schlaf-), repetitive Probleme $(p=0.011)$ und in Bezug auf die übergeordneten Skalen Internale Probleme
( $p<0.001)$ und Gesamtauffälligkeit ( $p<0.001)$. Die Interventionsgruppe zeigte in Bezug auf den SM-Schweregrad im Vergleich zur Kontrollgruppe eine Verringerung der SM-Symptome in allen Situationen (Schule: $p=0,003$, Familie: $p=0,018$, soziale Situation: $p=0,030$ ) und in der SMQ Gesamtpunktzahl ( $p<0,001$ ). Dies deuteten die Autorlnnen als Hinweis für die Wirksamkeit der Psychomotorik zur positiven Veränderungen des Lebensstils von Kindern mit selektivem Mutismus.

\section{Diskussion und Schlussfolgerung}

Die Studie von Alotaibi und KollegInnen (2017) wies darauf hin, dass ein bewegungstherapeutischer Ansatz die emotionale und physische Entwicklung von Kindern integrieren und fördern kann. Der komplette Datensatz mit $100 \%$ iger Rücklaufquote der Fragebögen und keinerlei Fehlzeiten der 60 Kinder über 24 Therapiestunden wirft jedoch die Frage nach sozialer Erwünschtheit bei der Beantwortung der Fragebögen auf, weswegen die Ergebnisse mit Vorsicht behandelt werden sollten. Esposito und KollegInnen (2016) verwendeten, laut ihrer Angabe, die italienische Version des $\mathrm{CBCL}$ und führten bei den Ergebnissen unter anderem die Subskalen „Social Relations“ und "School Competence“ auf. Diese sind jedoch weder in der aktuellen Version $\mathrm{CBCL} / 6-18$ noch in der ursprünglichen Version von 1991 enthalten.

Weitere RCTs mit Verblindung, großen Fallzahlen, Follow-Up-Tests und unterschiedlichen Klientengruppen (beispielsweise einer reinen Mädchengruppe) sind indiziert: Zukünftige Studien könnten die Langzeit-Wirksamkeit motorischer oder bewegungs-, ausdruckstherapeutischer Förderung auf die sozial-emotionale Entwicklung in Hinblick auf Lernstrategien oder pädiatrisch selektiven Mutismus überprüfen. Weitere Studien könnten der Frage nachgehen, welche spezifischen motorischen 
und bewegungstherapeutischen Interventionen die soziale und emotionale kindliche Entwicklung wirksam fördern können.

\section{Literatur}

Achenbach, T. M. (1991): Manual for the child behavior checklist / 4-18 and 1991 profile. University of Vermont Department of Psychiatry, Burlington

Alotaibi, A., Karkou, V., van der Linden, M., L., Irvine, L., M. C. (2017): Movement therapy programme with children with mild learning difficulties in primary schools in Saudi Arabia: Links between motion an emotion. In: Karkou, V., Oliver, S., Lycouris, S. (Hrsg.): The Oxford handbook of dance and wellbeing. Oxford, University Press, 479-492

Bergman, R. L., Keller, M. L., Piacentini, J., Bergman, A. J. (2008): The development and psychometric properties of the selective mutism questionnaire. Journal of Clinical Child \& Adolescent Psychology 37, 456-464

Esposito, M., Gimigliano, F., Barillari, M. R., Precenzano, F., Ruberto, M., Sepe, J., Barillari, U., Gimigliano, R., Militerni, R., Messina, G.,
Carotenuto, M. (2016): Pediatric selective mutism therapy: a randomized controlled trial. European Journal of Physical and Rehabilitation Medicine (e-pub. ahead of print)

Goodman, R., Ford, T., Simmons, H., Gatward, R., Goodman, R., Ord, T. F., Simmons, H. (2000): Using the Strengths and Difficulties Questionnaire (SDQ) to screen for child psychiatric disorders in a community sample. The British Journal of Psychiatry $177,534-539$

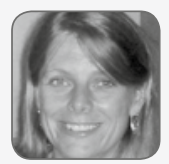

Dr. Iris Bräuninger, Dozentin / Forscherin (Hochschule für Heilpädagogik Zürich), Dozentin im Masterstudiengang Tanztherapie in Barcelona, Supervisorin / Ausbilderin / Lehrtherapeutin (BTD, ADMTE), Kestenberg Bewegungsnotatorin, Psychotherapie (ECP), private Praxis Bodensee.

\section{Dr. Iris Bräuninger} dancetherapy@mac.com und iris.braeuninger@hfh.ch 